\title{
Topological defects of optical anisotropy parameters caused by the screw dislocations of crystalline structure
}

\author{
Savaryn V., Vasylkiv Yu., Skab I. and Vlokh R. \\ Vlokh Institute of Physical Optics, 23 Dragomanov Street, 79005 Lviv, \\ Ukraine,vlokh@ifo.lviv.ua
}

Received: 30.09 .2015

\begin{abstract}
We have analyzed spatial distributions of optical birefringence and angle of optical indicatrix rotation caused by inhomogeneous mechanical stresses appearing due to structural screw dislocations in cubic and trigonal crystals. We have shown that accounting for even approximate boundary conditions leads to a physically sound result, zeroing of piezooptically induced birefringence in the vicinity of the dislocation core. Together with the availability of topological defect of the optical indicatrix orientation, this provides the exact conditions for generating singly charged optical vortices.
\end{abstract}

Keywords: screw dislocations, topological defects, piezooptic effect

PACS: $61.72 \mathrm{Ff}, 78.20 \mathrm{Fm}, 78.20 \mathrm{Hp}, 42.50 \mathrm{Tx}$

UDC: $535.5+544.022 .341 .1$

\section{Introduction}

Optical fibres, computer-generated holograms and spiral phase plates are commonly used for creating scalar field phase singularities of optical wave front [1-3], while vector field singularities can be induced with the aid of crystalline materials including bulk single crystals [4] or liquid crystals [5]. It is known that the phase singularities of the light wave front lead to appearance of optical vortices embedded into optical beams. Notice that the optical beams bearing nonzero orbital angular momentum have found various applications in different novel branches of optics. These are manipulation of micro-particles [6], reaching optical resolutions exceeding the diffraction limit [7], quantum cryptography, optical communications [8], etc.

Issuing from all of these considerations, earlier we have suggested a number of methods for creating the optical vortices using optical parametric effects induced by inhomogeneous external fields (electro- and piezooptic effects). This allows for operating the efficiency of spin-to-orbit angular momentum conversion [9-12]. Recently we have shown that dislocations of a crystalline structure can also produce the wave front dislocations $[13,14]$. The latter property can be used while detecting and studying the imperfections of a real crystalline structure. In particular, we have found that screw dislocations in crystals produce the topological defects (TD) of optical indicatrix (OI) orientation that have their strength equal to $1 / 2[13,14]$, while the edge dislocations produce the TD of OI orientation with the strength equal to unity [14].

In fact, the appearance of structural dislocations in crystals is accompanied by inhomogeneity of the mechanical stresses that surround a dislocation core. These stresses change the refractive indices of a material due to the piezooptic effect. As shown in Ref. [13], the transverse spatial distribution of piezooptically induced optical birefringence in the particular case of screw dislocations gives birth to the distribution of polarization states in the beam cross section, which is characterized with a so-called singular C-point.

Ukr. J. Phys. Opt. 2015, Volume 16, Issue 4 
In our earlier simulations performed in Ref. [15] we have used the analytical relations for the mechanical stress tensor components, which have been derived with no account for the boundary conditions. This imposes infinitely large mechanical stresses when the radial coordinate approaches that corresponding to the dislocation core. Of course, this result has no physical meaning. This has also led to infinitely large optical birefringence calculated in the vicinity of the dislocation core, which then generates a nonzero light intensity at the nucleus of optical vortex. Notice that this fact contradicts a principal property of any optical vortices: the phase value at the vortex nucleus should be undefined and the light intensity equal to zero. The present work is aimed at clarifying the facts mentioned above. This has been achieved while employing the refined analytical relations for the mechanical stress components obtained with taking the simplest boundary conditions into account.

\section{Results of analysis}

Below we will consider a screw dislocation appearing in the crystals that belong to the cubic (point symmetry groups $\mathrm{m} 3 \mathrm{~m}, 432$ and $\overline{4} 3 \mathrm{~m}$ ) and trigonal (point groups $3 \mathrm{~m}, 32$ and $\overline{3} \mathrm{~m}$ ) systems. In the case of trigonal crystals, the Burgers vector $\boldsymbol{b}$ and the dislocation line are parallel to [001] axis. For the cubic crystals they are parallel to [111] direction. For the sake of concreteness, we perform our analytical analysis for $\mathrm{LiNbO}_{3}$ (group $3 \mathrm{~m}$ ) and $\mathrm{NaCl}$ (group m3m) crystals.

Let us employ the general relations for the stress tensor components [16] derived for the half space $Z \geq 0$. Here a cylindrical cavity of radius $R_{0}$ is meant, which contains a screw dislocation with the Burgers vector parallel to the principal $Z$ axis. The appropriate analytical relations have been obtained with accounting for the boundary conditions $\left.\sigma_{i z}\right|_{z=0}=0$ and $\left.\sigma_{i r}\right|_{r=R_{0}}=0$, where $\varphi, r$ and $Z$ define a right-handed cylindrical coordinate system. They are as follows:

$$
\begin{aligned}
& \sigma_{r \varphi}=-\frac{G b}{2 \pi R_{0}} \frac{\tilde{r}^{2}}{\tilde{R}(\tilde{R}+\tilde{Z})^{2}}, \sigma_{z \varphi}=\frac{G b}{2 \pi R_{0}} \frac{\tilde{Z}}{\tilde{r} \tilde{R}}, \\
& \sigma_{r r}=\sigma_{\varphi \varphi}=\sigma_{z z}=\sigma_{z r}=0,
\end{aligned}
$$

where $\tilde{r}=\frac{r}{R_{0}}, \tilde{z}=\frac{Z}{R_{0}}, \tilde{R}=\sqrt{\tilde{r}^{2}+\tilde{z}^{2}}, R_{0}$ is the radius of the dislocation core, and $G$ the shear modulus.

Using the obvious links $\sigma_{4}=\sin \varphi \sigma_{r z}+\cos \varphi \sigma_{\varphi z}$ and $\sigma_{5}=\cos \varphi \sigma_{r z}-\sin \varphi \sigma_{\varphi z}$, one can rewrite Eqs. (1) in the Cartesian coordinate system $X Y Z$ :

$$
\sigma_{4}=\frac{G b}{2 \pi} \frac{Z X}{\left(X^{2}+Y^{2}\right) \sqrt{X^{2}+Y^{2}+Z^{2}}}, \quad \sigma_{5}=-\frac{G b}{2 \pi} \frac{Z Y}{\left(X^{2}+Y^{2}\right) \sqrt{X^{2}+Y^{2}+Z^{2}}} .
$$

At the condition $Z>>r$, Eqs. (2) are presented as [15]:

$$
\sigma_{4}=\frac{G b}{2 \pi} \frac{X}{X^{2}+Y^{2}}, \sigma_{5}=-\frac{G b}{2 \pi} \frac{Y}{X^{2}+Y^{2}} .
$$

Eqs. (3) have earlier been used in Refs. $[13,14]$. Here the stress tensor components tend to infinity when the $X$ and $Y$ coordinates are equal to zero. Using Eqs. (2), one can also obtain the dependences of the stress tensor component on the radial coordinate, either $X$ or $Y$.

In our simulations we have used the following parameters for the $\mathrm{NaCl}$ crystals: $G_{1}=G_{2}=G_{3}=11.8 \mathrm{GPa}$, and $\boldsymbol{b}=0.282 \mathrm{~nm}$. As seen from Fig. 1 , the stress tensor 
component $\sigma_{4}$ does not become infinitely large when the radial coordinate decreases, and the same is true of $\sigma_{5}$. Instead, the component $\sigma_{4}$ reaches a maximum at $r$ close to $R_{0}$ and becomes equal to zero at $r=R_{0}$.

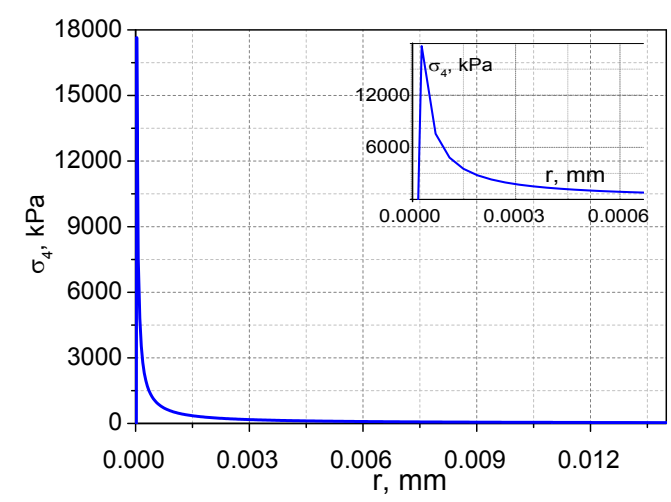

Fig. 1. Coordinate dependences of the stress tensor component $\sigma_{4}$, as calculated with Eqs. (2) for the case of a screw dislocation in $\mathrm{NaCl}$ crystals $\left(R_{0}=20 \mu \mathrm{m}\right.$ and $\left.Z=10 R_{0}\right)$. Insert shows dependence of the same stress tensor component calculated in the vicinity of dislocation core.

\subsection{NaCl crystals}

Using a standard matrix representation for the symmetric tensors, one can express the piezooptic effect in terms of induced changes in the optical impermeability coefficients $\Delta B_{i}$ [17]:

$$
\Delta B_{i}=B_{i}-B_{i}^{0}=\pi_{i j} \sigma_{j},
$$

where $\pi_{i j}$ is the fourth-rank piezooptic tensor, $B_{i}$ and $B_{i}^{0}$ the second-rank impermeability tensors written in the matrix form for the cases of stressed and free sample, respectively.

Let us consider the screw dislocation present in the crystalline structure of $\mathrm{NaCl}$, with the Burgers vector $\boldsymbol{b}$ being parallel to the [001] axis. We consider a light propagation along the same direction. Then the OI parameters read as

$$
\Delta B_{1}=\pi_{11} \sigma_{1}+\pi_{12} \sigma_{2}+\pi_{12} \sigma_{3}, \quad \Delta B_{2}=\pi_{12} \sigma_{1}+\pi_{11} \sigma_{2}+\pi_{12} \sigma_{3}, \quad \Delta B_{6}=\pi_{44} \sigma_{6} .
$$

Taking Eqs. (2) into account, we write out the birefringence and the angle of OI rotation as follows:

$$
\Delta n_{12}=-\frac{n^{3}}{2} \sqrt{\left(B_{1}-B_{2}\right)^{2}+4 \Delta B_{6}^{2}}=0, \quad \tan 2 \xi_{3}=\frac{2 \Delta B_{6}}{B_{1}-B_{2}}=0,
$$

where $n$ is the refractive index. As seen from Eqs. (6), there are no changes in the optical parameters whenever light propagates along the [001] axis.

Now let us consider the screw dislocation in $\mathrm{NaCl}$ with the $\boldsymbol{b}$ vector parallel to [111] and the case of light propagation along the $Z$ axis. For this aim we have to rewrite the both piezooptic and elastic compliance tensors in the new coordinate system $X^{\prime} Y^{\prime} Z^{\prime}$, in which $Z^{\prime}, Y^{\prime}$ and $X^{\prime}$ are parallel respectively to [111], [1110] and [112] directions. As a consequence, the OI parameters can readily be obtained:

$$
\Delta B_{1}^{\prime}=\pi_{14}^{\prime} \sigma_{4}+\pi_{15}^{\prime} \sigma_{5}, \quad \Delta B_{2}=-\pi_{14}^{\prime} \sigma_{4}-\pi_{15}^{\prime} \sigma_{5}, \quad \Delta B_{6}=-\pi_{15}^{\prime} \sigma_{4}+\pi_{14}^{\prime} \sigma_{5} .
$$

where

$$
\pi_{14}^{\prime}=-\pi_{15}^{\prime}=\frac{1}{3}\left(\pi_{11}-\pi_{44}-\pi_{12}\right)
$$

represents the piezooptic coefficient written in the $X^{\prime} Y^{\prime} Z^{\prime}$ system. Then the birefringence and the OI rotation angle are given by 


$$
\begin{aligned}
& \Delta n_{12}^{\prime}=-n^{3} \pi_{14}^{\prime} \frac{G_{1}^{\prime} b Z^{\prime}}{2 \pi\left(X^{\prime 2}+Y^{\prime 2}\right) \sqrt{X^{\prime 2}+Y^{\prime 2}+Z^{\prime 2}}} \sqrt{\left(X^{\prime}+Y^{\prime}\right)^{2}+4\left(X^{\prime}-Y^{\prime}\right)^{2}}, \\
& \tan 2 \xi_{[111]}=\frac{\sigma_{4}^{\prime}+\sigma_{5}^{\prime}}{\sigma_{4}-\sigma_{5}^{\prime}}=\frac{X^{\prime}-Y^{\prime}}{X^{\prime}+Y^{\prime}}=\frac{\cos \phi-\sin \phi}{\cos \phi+\sin \phi}=-\tan \left(\phi-\frac{\pi}{4}\right), \quad \xi_{[111]}=-\left(\frac{\phi}{2}-\frac{\pi}{8}\right) .
\end{aligned}
$$

Here $X^{\prime}=\rho \cos \phi, Y^{\prime}=\rho \sin \phi$ and $G_{1}^{\prime}=G_{2}^{\prime}=\frac{1}{S_{44}}$ [9], the refractive index at the light wavelength of $\lambda=632.8 \mathrm{~nm}$ is equal to $n=1.54$, and the piezo-optical coefficients are as follows: $\pi_{11}=1.27 \times 10^{-12} \mathrm{~m}^{2} / \mathrm{N}, \pi_{12}=2.58 \times 10^{-12} \mathrm{~m}^{2} / \mathrm{N}$ and $\pi_{44}=-0.84 \times 10^{-12} \mathrm{~m}^{2} / \mathrm{N}[18,19]$. Inserting Eqs. (2) into Eqs. (9), one can obtain the $X^{\prime} Y^{\prime}$ distributions of the birefringence and the angle of OI orientation. They are depicted in Fig. 2.
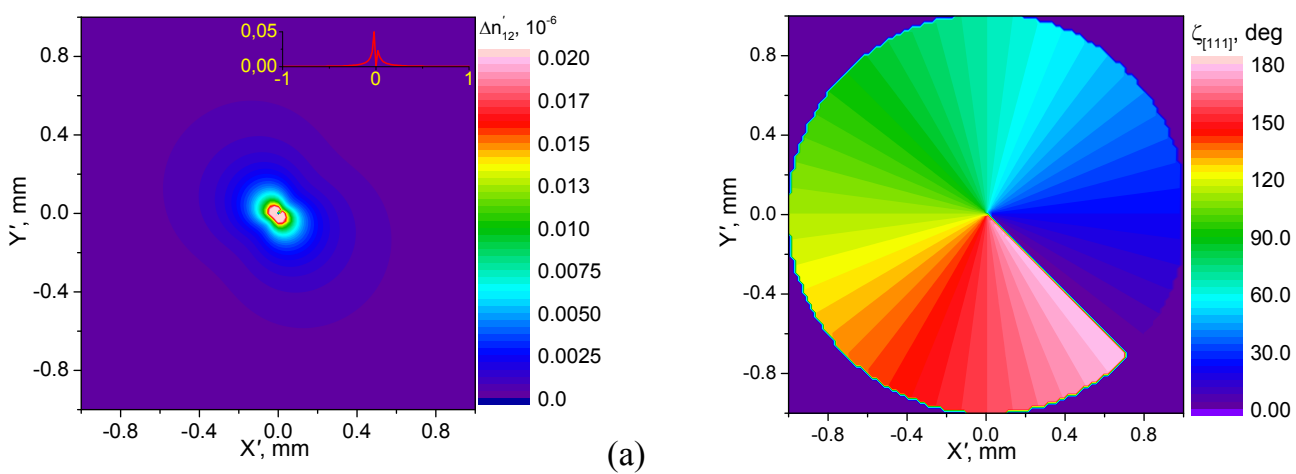

(b)

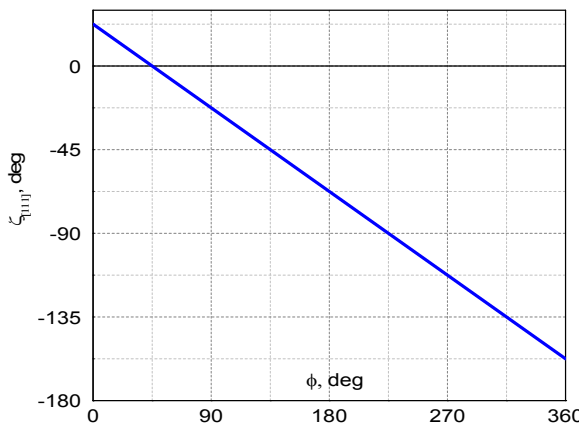

(a)

According to Fig. 2c, the angle of OI rotation depends linearly on the tracing angle. In other words, the screw structural dislocation in $\mathrm{NaCl}$ with the Burgers vector parallel to the [111] direction induces a pure screw dislocation of the light wave front, so that the strength of the TD equals to $-1 / 2$. Moreover, the piezooptically induced birefringence reaches a maximal magnitude at $r>R_{0}$ and become zero at $r \leq R_{0}$ (see Fig. 2a). This fact should yield zeroing of the intensity of light propagating through the dislocation core when a crystal sample is placed in between crossed circular polarizers. Therefore a singly charged optical vortex is generated. Hence, we have recovered the exact properties that correspond to the optical vortices even though only the approximate boundary conditions are used in our calculations.

\section{2. $\mathrm{LiNbO}_{3}$ crystals}

Now let us analyze the screw dislocation in the $\mathrm{LiNbO}_{3}$ crystals. We assume the Burgers vector parallel to the [001] axis and light propagation along the same direction. The increments of the 
optical impermeability tensor components in the $X Y$ plane can be written as

$$
\Delta B_{1}=\pi_{14} \sigma_{4}, \quad \Delta B_{2}=-\pi_{14} \sigma_{4}, \quad \Delta B_{6}=\pi_{14} \sigma_{5} .
$$

Then the birefringence and the angle of OI rotation become as follows:

$$
\Delta n_{12}=-\frac{n_{o}^{3}}{2} \pi_{14} \sqrt{\sigma_{4}^{2}+\sigma_{5}^{2}}, \quad \tan 2 \xi_{3}=\frac{\sigma_{5}}{\sigma_{4}}=\frac{Y}{X}=\tan \phi . .
$$

The analytical relations and the experimental data necessary for our further analysis of $\mathrm{LiNbO}_{3}$ are given by

$$
\begin{aligned}
& \qquad G_{1}=G_{2}=\frac{1}{S_{44}}, G_{3}=\frac{1}{2\left(S_{11}-S_{12}\right)}, \quad \mu_{1}=\mu_{2}=-\frac{S_{12}+S_{13}}{2 S_{11}}, \mu_{3}=-\frac{S_{13}}{S_{33}}, \quad \text { (12) } \\
& \pi_{14}=(0.875 \pm 0.071) \times 10^{-12} \mathrm{~m}^{2} / \mathrm{N} \quad[20], \quad S_{11}=5.831 \times 10^{-12} \mathrm{~m}^{2} / \mathrm{N}, \quad S_{33}=5.026 \times 10^{-12} \mathrm{~m}^{2} / \mathrm{N}, \\
& S_{12}=-1.150 \times 10^{-12} \mathrm{~m}^{2} / \mathrm{N}, \quad S_{13}=-1.452 \times 10^{-12} \mathrm{~m}^{2} / \mathrm{N} \quad \text { and } \quad S_{44}=17.10 \times 10^{-12} \mathrm{~m}^{2} / \mathrm{N} \quad[21], \\
& G_{1}=G_{2}=58.5 \mathrm{GPa} \text { and } G_{3}=71.6 \mathrm{GPa}, \quad \mu_{1}=\mu_{2}=0.223 \quad \text { and } \mu_{3}=0.289, \quad n_{o}=2.28647 \\
& (\lambda=632.8 \mathrm{~nm}), R=10^{-3} \mathrm{~m}, \text { and } R_{0}=20 \mu \mathrm{m} \text {. Using Eqs. (2) and Eqs. (12), one can derive the } X Y \\
& \text { distributions of the birefringence and the angle of OI orientation, which appear in the case when } \\
& \text { the Burgers vector is parallel to [001] and the light propagates along the } Z \text { axis. }
\end{aligned}
$$
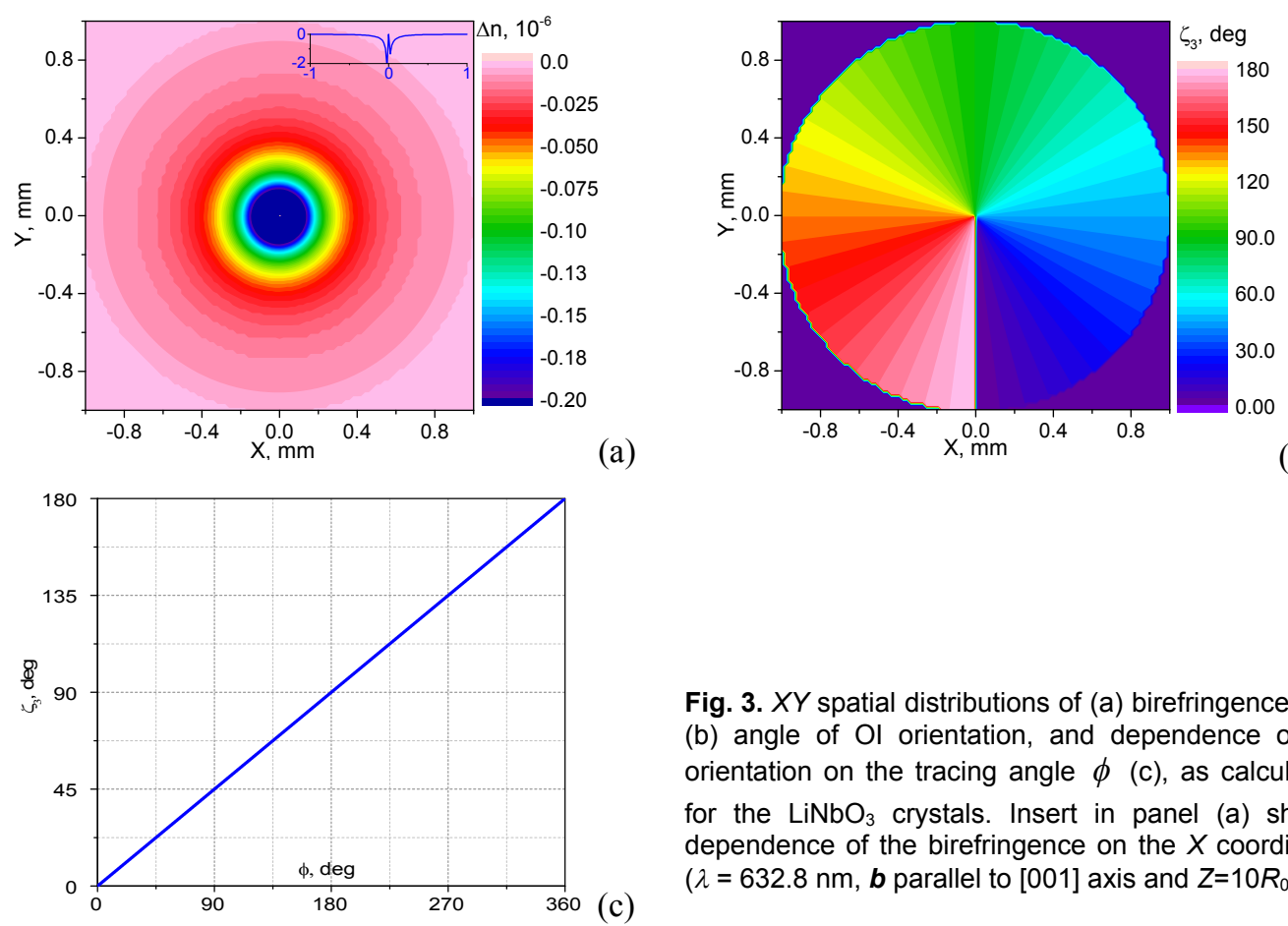

Fig. 3. $X Y$ spatial distributions of (a) birefringence and (b) angle of Ol orientation, and dependence of OI orientation on the tracing angle $\phi$ (c), as calculated for the $\mathrm{LiNbO}_{3}$ crystals. Insert in panel (a) shows dependence of the birefringence on the $X$ coordinate $\left(\lambda=632.8 \mathrm{~nm}, \boldsymbol{b}\right.$ parallel to [001] axis and $\left.Z=10 R_{0}\right)$.

As seen from Fig. 3c, the OI rotation depends linearly on the tracing angle. Hence, the screw dislocation in the crystalline structure of $\mathrm{LiNbO}_{3}$ characterized with the Burgers vector parallel to the [001] axis induces a pure screw dislocation of the light wave front, which corresponds to the TD strength equal to $+1 / 2$ (Fig. $3 \mathrm{~b}$ ). Since the mechanical stress components are equal to zero at $r \leq R_{0}$, the optical birefringence under these conditions is also zero (see Fig. 3a). In other words, placing either $\mathrm{LiNbO}_{3}$ or $\mathrm{NaCl}$ crystalline samples in between crossed circular polarizers would produce a canonical optical vortex with the topological charge equal to \pm 1 . 


\section{Conclusions}

We have derived analytically the changes in the spatial distributions of optical anisotropy parameters (the optical birefringence and the angle of OI rotation), which are induced by the mechanical stresses caused by the screw dislocations of the crystalline structure. As examples, we have simulated the cases of canonical $\mathrm{NaCl}$ and $\mathrm{LiNbO}_{3}$ crystals that belong respectively to the cubic and trigonal systems. The analytical relations for the stress tensor components have been employed, which account for the approximate boundary conditions.

We have shown that availability of the screw dislocations of crystalline structure in the trigonal materials induces the pure screw dislocation of the light wave front, whenever the Burgers vector is parallel to the [001] axis and the light propagates along the same direction. Then the strength of the TD is equal to $+1 / 2$. In case of the cubic crystals with the screw dislocation, we have arrived at the pure screw dislocation of the light wave front appearing when the Burgers vector and the light wave vector are parallel to the [111] direction. Then the strength of the TD is equal to $-1 / 2$. Notice that the TD strengths characteristic for the different crystalline systems considered here have the opposite signs ("minus" for the cubic crystals and "plus" for the trigonal ones).

Finally, we have shown that the optical birefringence is zero under the condition $r \leq R_{0}$ because the mechanical stresses are then equal to zero. As a result, placing the samples of $\mathrm{LiNbO}_{3}$ or $\mathrm{NaCl}$ in between the crossed circular polarizers should result in a canonical optical vortex, with the charge equal to \pm 1 . This implies that the screw dislocations of the crystalline structure can be detected using an optical microscope and relevant crystal samples placed in between the crossed circular polarizers.

\section{References}

1. Heckenberg N R, McDuff R, Smith C P and White A G, 1992. Generation of optical phase singularities by computer-generated holograms. Opt. Lett. 17: 221-223.

2. Oemrawsingh S S R, van Houwelingen J A W, Eliel E R, Woerdman J P, Verstegen E J K, Kloosterboer J G and Hooft G W, 2004. Production and characterization of spiral phase plates for optical wavelengths. Appl. Opt. 43: 688-694.

3. Volyar A V, 2002. Fiber singular optics. Ukr. J. Phys. Opt. 3: 69-96.

4. Desyatnikov A, Fadeyeva T A, Shvedov V G, Izdebskaya Y V, Volyar A V, Brasselet E, Neshev D N, Krolikowski W and Kivshar Y S, 2010. Spatially engineered polarization states and optical vortices in uniaxial crystals. Opt. Expr. 18: 10848-10863.

5. Marrucci L, 2008. Generation of helical modes of light by spin-to-orbital angular momentum conversion in inhomogeneous liquid crystals. Mol. Cryst. Liq. Cryst. 488: 148-162.

6. Grier D G, 2003. A revolution in optical manipulation. Nature. 424: 810-816.

7. Qiwen Zhan, 2006. Properties of circularly polarized vortex beams. Opt. Lett. 31: 867-869.

8. Molina-Terriza G, Vaziri A, Rehácek J, Hradil Z and Zeilinger A, 2004. Triggered qutrits for quantum communication protocols. Phys. Rev. Lett. 92: 167903.

9. Skab I, Vasylkiv Yu, Smaga I and Vlokh R, 2011. Spin-to-orbital momentum conversion via electrooptic Pockels effect in crystals. Phys. Rev. A. 84: 043815.

10. Skab I, Vasylkiv Y, Savaryn V and Vlokh R, 2011. Optical anisotropy induced by torsion stresses in $\mathrm{LiNbO}_{3}$ crystals: appearance of an optical vortex. J. Opt. Soc. Amer. A. 28: 633640.

11. Skab I, Vasylkiv Yu, Zapeka B, Savaryn V and Vlokh R, 2011. On the appearance of singularities of optical field under torsion of crystals containing three-fold symmetry axes. 
J. Opt. Soc. Amer. A. 28: 1331-1340.

12. Skab I, Vasylkiv Yu and Vlokh R, 2012. Induction of optical vortex in the crystals subjected to bending stresses. Appl. Opt. 51: 5797-5805.

13. Savaryn V, Vasylkiv Yu, Krupych O, Skab I and Vlokh R, 2013. Polarization singularities of optical fields caused by structural dislocations in crystals. J. Opt. 15: 044023.

14. Savaryn V, Vasylkiv Yu, Krupych O, Skab I and Vlokh R, 2015. Corrigendum: Polarization singularities of optical fields caused by structural dislocations in crystals (J. Opt. 2013, 15 044023). J. Opt. 17: 089501.

15. Friedel J, Dislocations. Oxford: Pergamon Press (1964).

16. Sheinerman A. G. and Gutkin M. Yu, 2003. Elastic fields of a screw super-dislocation with a hollow core (pipe) perpendicular to the free crystal surface. Phys. Solid State. 45: 1694-1700.

17. Narasimhamurty T S, Photoelastic and electrooptic properties of crystals. New York: Plenum Press (1981).

18. Hellwege K-H and Hellwege A M, 1979. Landolt-Börnstein numerical data and functional relationships in science and technology, New Series, Group III: Crystal and solid state physics, 11: Elastic, piezoelectric, pyroelectric, piezooptic, electrooptic constants and nonlinear susceptibilities of crystals (Berlin: Springer-Verlag)

19. http://www.optotl.ru/mat/NaCl

20. Vasylkiv Yu, Savaryn V, Smaga I, Skab I and Vlokh R, 2011. On determination of sign of the piezo-optic coefficients using torsion method. Appl. Opt. 50: 2512-2518.

21. Weis R S and Gaylord T K, 1985. Lithium niobate: Summary of physical properties and crystal structure. Appl. Phys. A. 37: 191-203.

Savaryn V., Vasylkiv Yu., Skab I. and Vlokh R. 2015. Topological defects of optical anisotropy parameters caused by the screw dislocations of crystalline structure. Ukr.J.Phys.Opt. 16: 171-177.

Анотація. У роботі проаналізовано просторові розподіли оптичного двозаломлення $і$ кута повороту оптичної індикатриси, спричинені механічними напруженнями, щзо виникають внаслідок існування структурних гвинтових дислокацій у кубічних $i$ тригональних кристалах. Показано, що врахування навіть наближених граничних умов приводить до занулення n'єзооптично індукованого двозаломлення в околі сериевини дислокації. Разом із існуванням топологічного дефекту орієнтації оптичної індикатриси, ие забезпечує точні умови для генерації оптичних вихорів із зарядом, щзо дорівнює одиниці.

Ukr. J. Phys. Opt. 2015, Volume 16, Issue 4 